\title{
Ringversuche für Steroidhormonbestimmungen: Richtigkeit und Präzision der Analysenergebnisse
}

\author{
Von G. Röhle \\ Institut für Klinische Biochemie der Universität Bonn, \\ U. Voigt
}

Institut für Medizinische Statistik, Dokumentation und Datenverarbeitung der Universität Bonn,

L. Siekmann und $H$. Breuer $\dagger^{1}$ )

Institut für Klinische Biochemie der Universität Bonn

(Eingegangen am 26. April/9. November 1982)

Zusammenfassung: In den Jahren 1977 bis 1981 wurden in Zusammenarbeit mit der Deutschen Gesellschaft für Klinische Chemie 14 Ringversuche für Steroidhormonbestimmungen durchgeführt. Hierbei konnten von den teilnehmenden Laboratorien jeweils folgende Steroide analysiert werden: Aldosteron, Cortisol, Östradiol-17 $\beta$, Östriol, Progesteron und Testosteron. Anhand der Resultate wurde untersucht, ob im Laufe der Zeit eine Verbesserung dẹr Richtigkeit bzw. der Präzision der Bestimmungen eingetreten ist und in welchem Mảße die Bestimmungen von den Eigenschaften des Probenmaterials abhängen. Eine deutliche Verbesserung der Richtigkeit der Analysenergebnisse war nur für Östradiol-17 $\beta$ festzustellen. Für Aldosteron und Cortisol wurden in „Pool-Plasma“ Werte ermittelt, deren Mediane signifikant über den definitiven Werten lagen.

Eine Verbesserung der Präzision konnte besonders bei Östradiol-17 $\beta$, in geringerem Maße auch bei Cortisol und Östriol beobachtet werden. Die Art des Probenmaterials - Plasma, das nur die zu analysierenden Hormone enthielt einerseits, und „Pool-Plasma“, das zusätzlich alle endogenen Hormone enthielt, andererseits hatten keinen Einfluß auf die Präzision der Resultate aus verschiedenen Laboratorien.

Niedrige Könzentrationen der einzelnen Steroide führten - aufgrund des methodischen Prinzips von Radiọimmunoạssays = in fast allen Fällen zu einer schlechteren Präzision der Werte aus verschiedenen Laboratorien. Die Richtigkeit der Analysenwerte wurde nur bei Aldosteron und Östradiol-17 $\beta$ durch niedrige Konzentrationèn erheblich beeinträchtigt: die Mediane waren hierbei teilweise zweimal so hoch wie die definitiven Werte.

Quality control surveys for the determination of steroid hormones: Accuracy and precision of analysis results

Summary: In the years 1977 to 1981,14 quality-control surveys for the determination of steorid hormones were performed in cooperation with the Deutsche Gesellschaft für Klinische Chemie. Hereby the laboratories participating could in each case analyze the following steroids: aldosterone, cortisol, oestradiol-17 $\beta$, oestriol, progesterone, and testosterone. In the light of the results an investigation was made as to whether, in the course of time, an improvement in the accuracy or in the precision of the determinations had been attained, and to what extent the determinations depend on the qualities of the test material. A clear improvement in the

1) Prof. Dr. H. Breuer, member of the Editorial Board of our journal died August 20, 1982. 
accuracy of the results of the analyses could only be ascertained for oestradiol-17 $\beta$. For aldosterone and cortisol, values were found in pool-plasma whose medians were significantly above the definitive values.

An improvement in precision could be noted especially with oestradiol-17 $\beta$ and to lesser degrees with cortisol and oestriol. The kind of test material - plasma which contained only the hormones to be analyzed on the one hand, and, on the other hand, pool-plasma, which also contained all endogenous hormones = had no influence on the precision of the results from various laboratories.

Low concentrations of the individual steroids led - on the basis of the methodological principle of radioimmunoassays - in almost all cases to a reduced interlaboratory precision in regard to values. The accuracy of the analysis values was considerably impaired only with aldosterone and oestradiol-17 $\beta$ by low concentra= tions: the medians here were in part twice as high as the definitive values.

\section{Einführung}

Innerhalb des letzten Jahrzehnts wurden nahezu überall in der Welt Maßnahmen zur internen und externen Qualitätskontrolle quantitativer klinisch-chemischer Analysen eingeführt. Große Erwartungen hinsichtlich einer Vèrbesserung der Analytik setzte man insbesondere in die Qualitätskontrolle radiound enzymimmunologischer Bestimmungen von Hormonen, da viele Hinweise dafür sprachen, daß die Zuverlässigkeit der mit diesen Analysentechniken ermittelten Ergebnisse nicht in ausreichendem Maße gegeben war (1). Dies galt in erster Linie für die Vergleichbarkeit von Analysenergebnissen aus verschiedenen Laboratorien.

Es ist grundsätzlich festzustellen, daß die Maßnahmen der Qualitätskontrolle keinen unmittelbaren Einfluß auf die Zuverlässigkeit der Analysenergebnisse haben können; von Resultaten der Qualitätskontrolle sind lediglich Impulse zur Verbesserung der Analytik zu erwarten. Da eine sehr große Zahl verschiedener Fehlerquellen die Zuverlässigkeit von Analysenergebnissen beeinträchtigen können, ist es notwendig, die wichtigsten Fehlerquellen zu erkennen, um Ansatzpunkte für eine Verbesserung der Analytik zu erhalten: Von den bekannten Verfahren zur externen Qualitätskontrolle von Hormonbestimmungen ist unter diesem Aspekt das von Scriba et al. entwickelte Ringversuchsmodell $(2-5)$ besonders geeignet. Es hat allerdings den Nachteil, daß es wegen seiner sehr komplizierten Versuchanordnung mit jeweils etwa 20 Proben für ein einzelnes Hormon für eine regelmäßige Kontrolle aller wichtigen Hormonbestimmungen kaum praktikabel ist.

Seit 1976 wurden in Zusammenarbeit mit der Deutschen Gesellschaft für Klinische Chemie regelmäßig Ringversuche für Hormonbestimmungen durchgeführt, die auf einem einfacheren Modell beruhen (611). Úber die Ergebnisse soll im folgenden berichtet werden. Den teilnehmenden Laboratorien wurden zu jedem Ringversuch jeweils zwei Kontrollproben mit unterschiedlichen Konzentrationen an Steroidhormonen zur Verfügung gestellt. Jede Kontrollprobe enthielt u.a. sechs Steroidhormone, deren Konzentrationen vor Beginn der Ringversuche mit der massenspektrometrischen Isotopenverdünnungsmethode festgestellt wurden (12).

Weiterhin konnten in jedem Ringversuch sechs Peptidhormone sowie Thyroxin und Triiodthyronin bestimmt werden; die Ergebnisse dieser Bestimmungen sind jedoch nicht Gegenstand der vorliegenden Untersuchung.

Anhand der Resultate aus den 14 Ringversuchen wird in der vorliegenden Arbeit untersucht, ob im Laufe der Zeit eine Verbesserung der Analytik eingetreten ist und in welchem Maße Richtigkeit und Präzision der Ergebnisse der teilnehmenden Laboratorien von den Eigenschaften des Probenmaterials abhängen.

\section{Material und Methoden}

Kontrollproben

Ausgangsmaterial für alle verwendeten Kontröllproben war gepooltes Humanplasma bzw. Humanserum. Hieraus wurdèn inisgesamt 18 Chargen von Probenmaterial mit unterschiedlichẹn Konzentrationen der Analyte hergestellt.

Bei acht Plasmaproben wurden die endogenen Steroide durch Adsorption an Aktivkohle entfernt (gestripptes Plasma); anschließend wưrden die Analyte in zuvor féstgelegten Mengen zugesetzt. Fünf Plasma-Proben und fünf Serum=Proben enthielten alle endogenen Plasmabestandteile; die Konzeñtrationen der Analyte wurden jedoch z.T. đụrch Zusatz vón Reinsubstanzen erhöht (Pool-Proben). Nach Homogenisierung einer Probe wurde das Material in Portionen zu je $5 \mathrm{ml}$ in Glasgefäße abgeffullt, lyophilisiert und verschlossen.

\section{Definitive Werte}

Jeweils sechs Abfüllungen einer Probe wurden mit je $5 \mathrm{ml}$ bidestilliertem Wasser rekonstituiert. Im rekonstituierten Plasma bzw. 
Serum wurden die Konzentrationen der Steroide Aldosteron, Cortisol, Ostradiol-17 $\beta$, Ostriol, Progesteron und Testosteron mit der massenspektrometrischen Isotopenverdünnungsanalyse bestimmt (12).

\section{Ringversuche}

In 14 Ringversuchen konnten von den teilnehmenden Laboratorien jeweils sechs verschiedene Steroidhormone bestimmt werden: Aldosteron, Cortisol, Ostriol, Ostradiol-17ß, Progesteron und Testosteron.

Bei jedem Ringversuch erhielt jedes teilnehmende Laboratorium Abfüllungen von zwei verschiedenen Proben zur quantitativen Bestimmung der Steroidhormone. Die Teilnehmer bestimmten die Steroidkonzentrationen mit radioimmunologischen oder vergleichbaren Routinemethoden. Für die Untersuchung stand jeweils ein Zeitraum von einem Monat zur Verfügung.

Nach zwei Pilot-Studien im Jahre 1976, bei denen jeweils nur drei Steroidhormone analysiert wurden, konnten von 1977 bis 1981 insgesamt 14 Ringversuche mit jeweils sechs Steroiden durchgeführt werden. Die Art des Probenmaterials, die Kennzeichnung der Proben und deren Zuordnung zu den einzelnen Ringversuchen sind in der Tabelle 1 verzeichnet. Die Proben G, H, J, K, 4, $7,8,10$ und 11 wurden in verschiedenen Ringversuchen eingesetzt.

\section{Auswertung und statistische Methoden}

In die Auswertung wurden alle Teilnehmerergebnisse $\mathrm{zu}$ den Ringversuchen Nr. 3-16 einbezogen.

\section{Variabilität}

Die Analysenergebnisse für die einzelnen Bestandteile der Ringversuchsproben sind nicht normalverteilt. Deshalb wird als $\mathrm{MaB}$ für die Streuung unter Vergleichsbedingungen in Analogie zum parametrischen Variationskoeffizienten eine nichtparametrische Größe bewählt, die als die Hälfte der Differenz zwischen dem Wert der $16 \%$ Perzentile und dem Wert der $84 \%$ Perzentile, ausgedrückt in Prozent des Medianwertes, definiert und mit relativer Variabilität bezeichnet wird.

$\begin{aligned} & \text { Relative } \\ & \text { Variabilität }\end{aligned}=\frac{(84 \% \text { Perzentile }-16 \% \text { Perzentile }) \cdot 100}{2 \cdot \text { Median }}, \%$

Ein solches Vorgehen führt bei den hier vorliegenden asymmetrischen Verteilungen naturgemäß zu einem gewissen Informationsverlust, erlaubt es aber, die gewohnte Darstellungsweise beizuhalten, ohne die durch ein parametrisches Maß sich ergebende Verfälschung in Kauf nehmen zu müssen.

\section{Unrichtigkeit}

Für jede Probe und jeden Ringversuch wurde die Unrichtigkeit des Gesamtergebnisses für Ostradiol- $17 \beta$ als relative Abweichung des Medians der Analysenergebnisse vom definitiven Wert ermittelt.

Relative Unriçhtigkeit $=\frac{(\text { Median }- \text { definitiver Wert }) \cdot 100}{\text { definitiver Wert }} \%$

\section{Ergebnisse und Diskussion}

An den Ringversuchen nahmen bis zu 200 Laboratorien aus der Bundesrepublik Deutschland und 70 aus anderen europäischen Ländern teil. In der Tabelle 1 sind für jeden Ringversuch die Zahl der Teil- nehmer angegeben, für jeden Analyten die Zahl der Analysenergebnisse mit dem zugehörigen Median und der definitive Wert.

Die Zuverlässigkeit der Ergebnisse von Steroidhormonbestimmungen in Ringversuchen ist - wie bei allen biochemischen Analysen - von mehreren Faktoren abhängig. Neben den analytischen Voraussetzungen innerhalb eines Laboratoriums und der Sorgfalt des Analytikers haben die Eigenschaften des Untersuchungsmaterials offensichtlich einen wesentlichen Einfluß auf das Ergebnis.

Nach den vorliegenden Daten hängen die Richtigkeit und die Präzision der Ringversuchsergebnisse aus verschiedenen Laboratorien u.a. ab von der Konzentration des zu bestimmenden Bestandteils und von der Anwesenheit endogener niedermolekularer Störsubstanzen, die bei den immunologischen Bestimmungsverfahren interferieren. Unter den gegebenen Versuchsbedingungen ist die Summe aller variablen Faktoren, welche die Analytik beeinflussen, so groß, daß strenge quantitative Zusammenhänge zwischen Einflußgröße und Ergebnis nicht festzustellen sind. Qualitative Zusammenhänge und Tendenzen lassen sich jedoch deutlich erkennen.

Als Kriterien für die Zuverlässigkeit der Analysenergebnisse in den Ringversuchen werden folgende Kenngrößen verwendet:

1. Die Abweichung des Medians aller Analysenergebnisse eines Ringversuchs für einen Analyten vom definitiven Wert (Richtigkeit).

2. Die Präzision der Analysenwerte aus verschiedenen Laboratorien.

Eine Verbesserung der Zuverlässigkeit der Ergebnisse im Laufe der Zeit ist nur bei Östradiol-17 $\beta$ sowohl für die Richtigkeit als auch für die Präzision - deutlich zu erkennen. Allerdings waren die Resultate für diesen Analyten zunächst besonders ungünstig. Die Verbesserung der Präzision trat im Jahre 1979 sprunghaft ein: für die Probe ,J“ lag die relative Variabilität der Werte für Östradiol-17 $\beta$ im 7. Ringversuch bei $57 \%$; im 8 . Ringversuch dagegen bei $28 \%$ (Abb. 1).

Die Abbildung 1 zeigt auf den Ordinaten die relative Variabilität und die relative Unrichtigkeit des $\mathrm{Me}$ dians aller Werte für Östradiol-17ß; die Skala der Abszisse gibt die Reihenfolge der Ringversuche wieder. Die unterschiedliche Markierung der Säulen der Histogramme zeigt an, ob gestripptes Plasma oder Pool-Proben verwendet wurden. Zusätzlich sind diejenigen Proben gekennzeichnet, in denen die Ostradiol-17 $\beta$ Konzentration kleiner als $0,5 \mathrm{nmol} / \mathrm{l}$ war. 


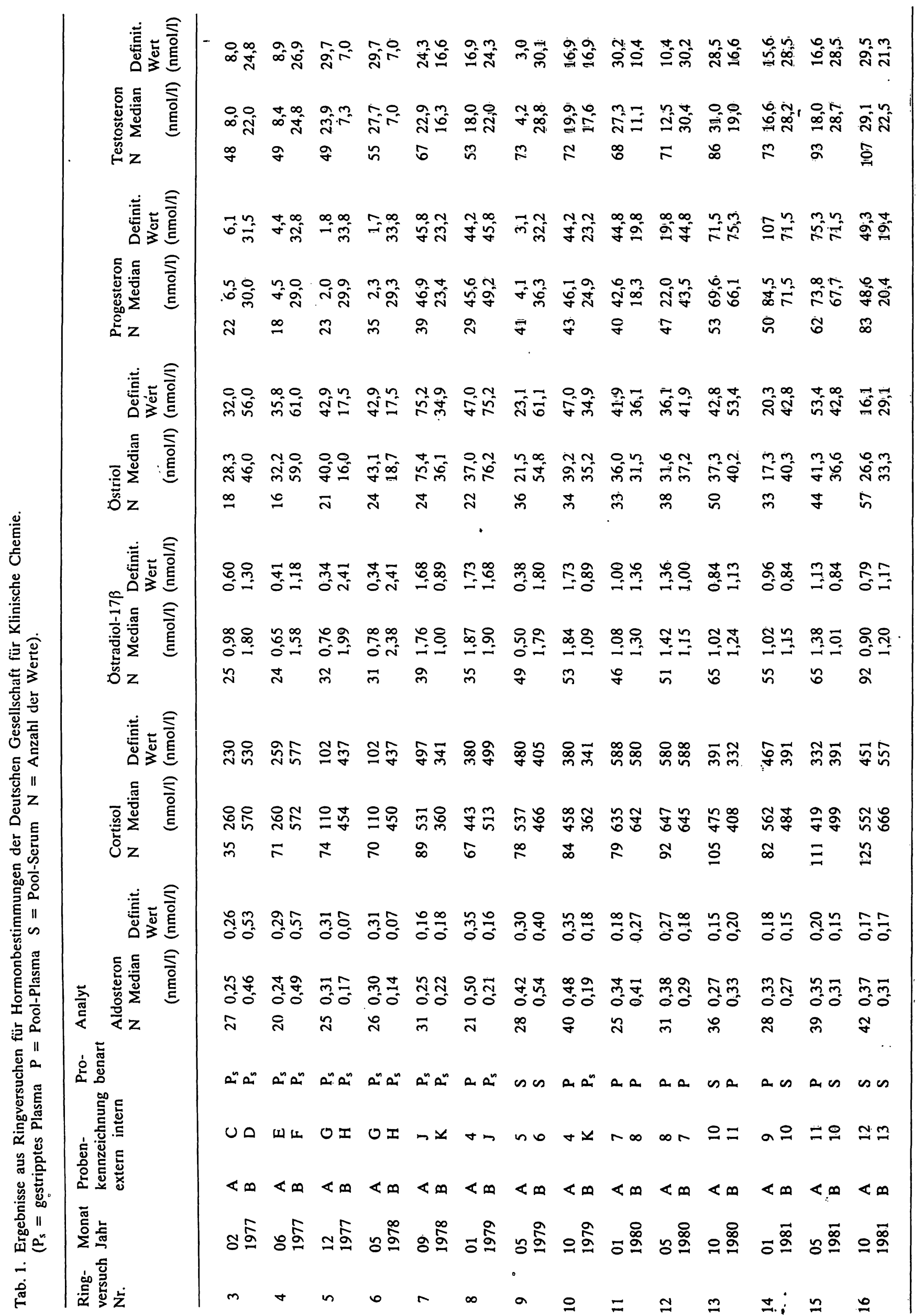




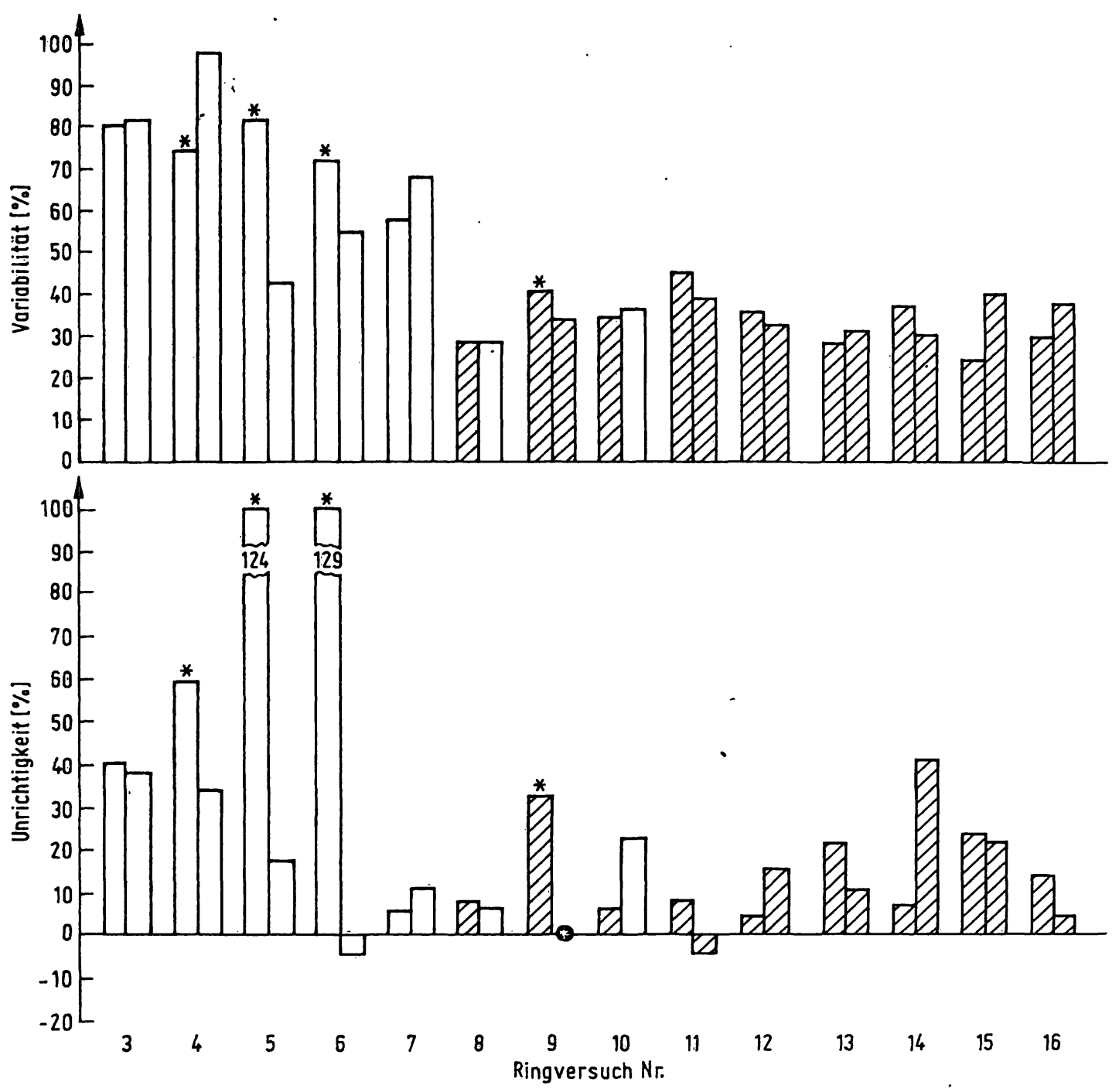

Abb. 1. Relative Variabilität und relative Unrichtigkeit der Analysenergebnisse für Ostradiol-17 $\beta$ aus den Ringversuchen 3-16 (1977-1981). $\square$ Gestripptes Plasma; Pool-Plasma; $\star$ Konzentration $<5$ nmol/l.

In diesen Fällen ist die sehr große Abweichung der Analysenergebnisse vom definitiven Wert besonders auffallend.

Unter Berụ̈cksichtigung der unterschiedlichẹn Eigenschaften des Probenmaterials - Konzentration der Analyte einerseits und gestrippte Proben bzw. Pool-Proben anderèrseits - ist auch für Cortisol und Östriol eine Tendenz zu einer Verbesserung der Prä- zision der Werte im Laufe der Zeit zu erkennen. Als Beispiel hierzu ist der zeitliche Verlauf der Variabilitạ̈t für die Bestimmung von Östriol dargestellt (Abb. 2).

In den Abbildungen 3-8 sind für alle untersuchten Steroidhormone einerseits die Mediane der verschiedenen Ringversuchsergebnisse den definitiven Werten gegenübergestellt und andererseits die ent-

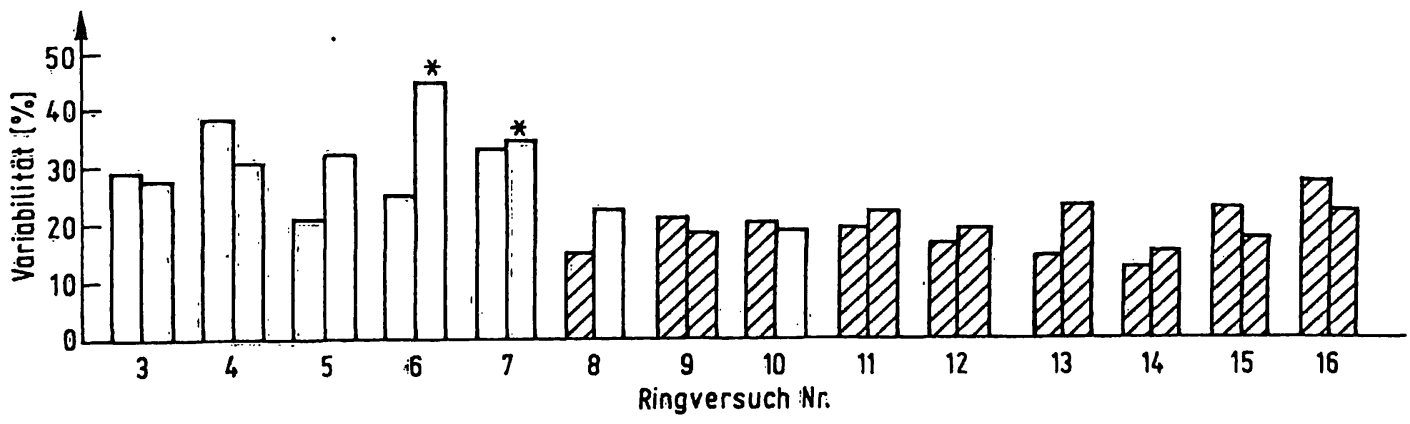

Abb. 2. Relative Variabilität der Analysenergebnisse für Ostriol aus den Ringversuchen 3-16 (1977-1981).

$\square$ Gestripptes Plasma; Pool-Plasma; $\star$ Konzentration $<20 \mathrm{nmol} / \mathrm{h}$. 
sprechenden Werte für die Variabilität - ebenfalls gegenüber den gegebenen Konzentrationen.

Offensichtlich ist der Stand der Analytik für die einzelnen Steroidhormone sehr unterschiedlich; auch ist der Effekt, der durch die Art des Probenmaterials und die Höhe der Analyt-Konzentration hervorgerufen wird, nicht einheitlich.

Die Zuverlässigkeit der Ergebnisse für Aldosteron (Abb. 3) ist sehr schlecht. Die relative Variabilität liegt für mittlere Konzentrationen bei etwa $45 \%$, steigt aber mit abnehmender Konzentration steil an. Die Art des Probenmaterials hat hier - ebenso wie bei den übrigen Steroiden - anscheinend keinen Einfluß auf die Variabilität der Werte.

Der Vergleich der von den Ringversuchsteilnehmern ermittelten Aldosteronkonzentrationen mit den de-
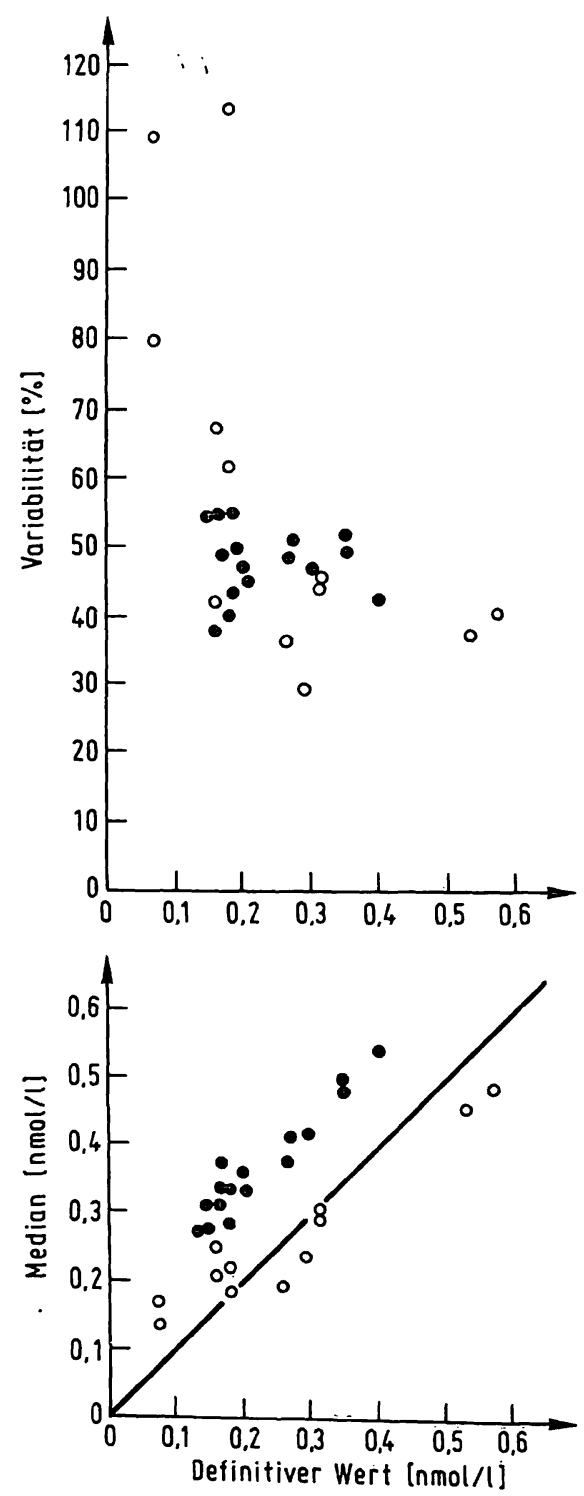

Abb. 3. Relative Variabilität (oben) der Ergebnisse aus 14 Ringversuchen und die Werte der Mediane (unten) - jeweils gegenüber den gegebenen Konzentrationen von Aldosteron. O Gestripptes Plasma; - Pool-Plasma. finitiven Werten zeigt für gestripptes Plasma nur bei niedrigen Konzentrationen besonders große Differenzen. Hingegen liegen die Werte für Pool-Proben fast immer um etwa $0,15 \mathrm{nmol} / \mathrm{l}$ über den definitiven Werten; vermutlich führen Kreuzreaktionen mit den im Pool-Plasma enthaltenen endogenen Steroiden zu diesen nahezu konstanten Abweichungen.

Die Zuverlässigkeit der Bestimmungen von Cortisol (Abb. 4) ịst wesentlich besser. Die Variabilität liegt meistens um $20 \%$, und die Richtigkeit ist in fast allen Fällen gut. Dennoch sind Pạrallelen zu dem bei Aldosteron beobachteten Phänomen zu erkennnen: die größere Variabilität bei niedrigen Konzentrationen und erhöhte Werte der Mediane (etwa $60 \mathrm{nmol} / \mathrm{l}$ ) bei Pool-Proben.

Schwieriger zu erkennen sind die Zusammenhänge zwischen den Eigenschaften der Proben und den Resultaten für Östradiol-17 $\beta$ (Abb. 5). Es scheint, daß die Bestimmung in Pool-Próben mit wesentlich höherer Präzision möglich ist. Da diese Art von Material jedoch erst ab dem 8. Ringversuch eingesetzt wurde. (Abb. 1; Tab. 1), dürfte hauptsächlich die Verbesserung der Analytik die relativ besseren $\mathrm{Re}-$ sultate verursacht haben; ab dem 8 . Ringversuch la-
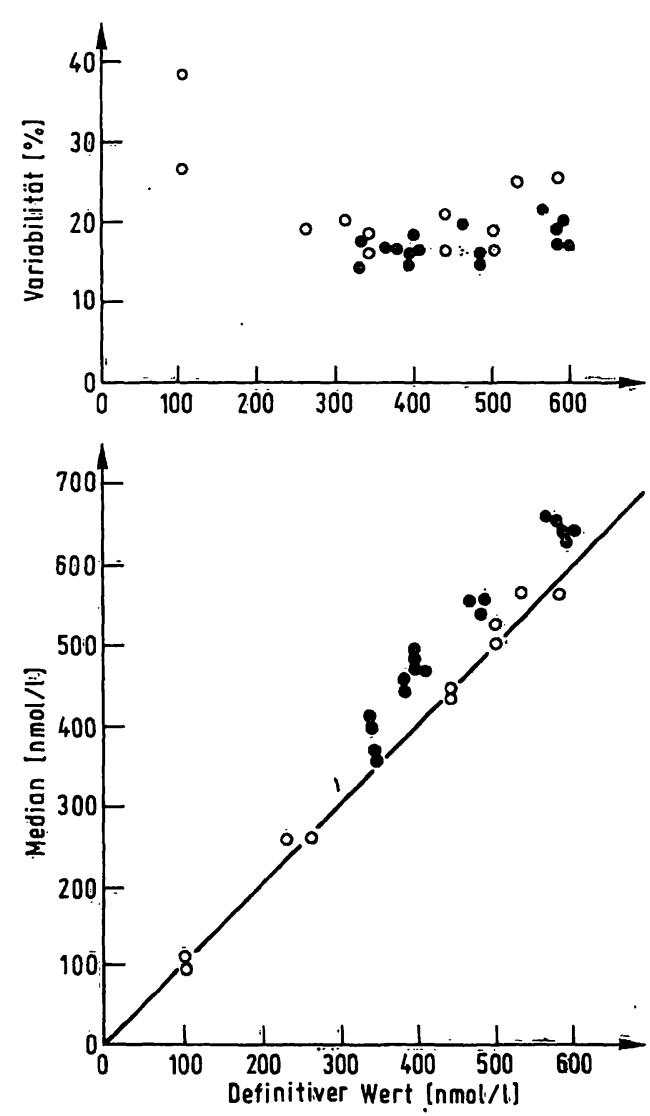

Abb. 4. Relative Variabilität (oben) der Ergebnisse aus 14 Ringversuchen und die Werte der Mediane (unten). - jeweils gegenüber deń gegebenen Konzentrationen von Cortisol. O Gestripptes Plasma; 1 Popl-Plasma. 

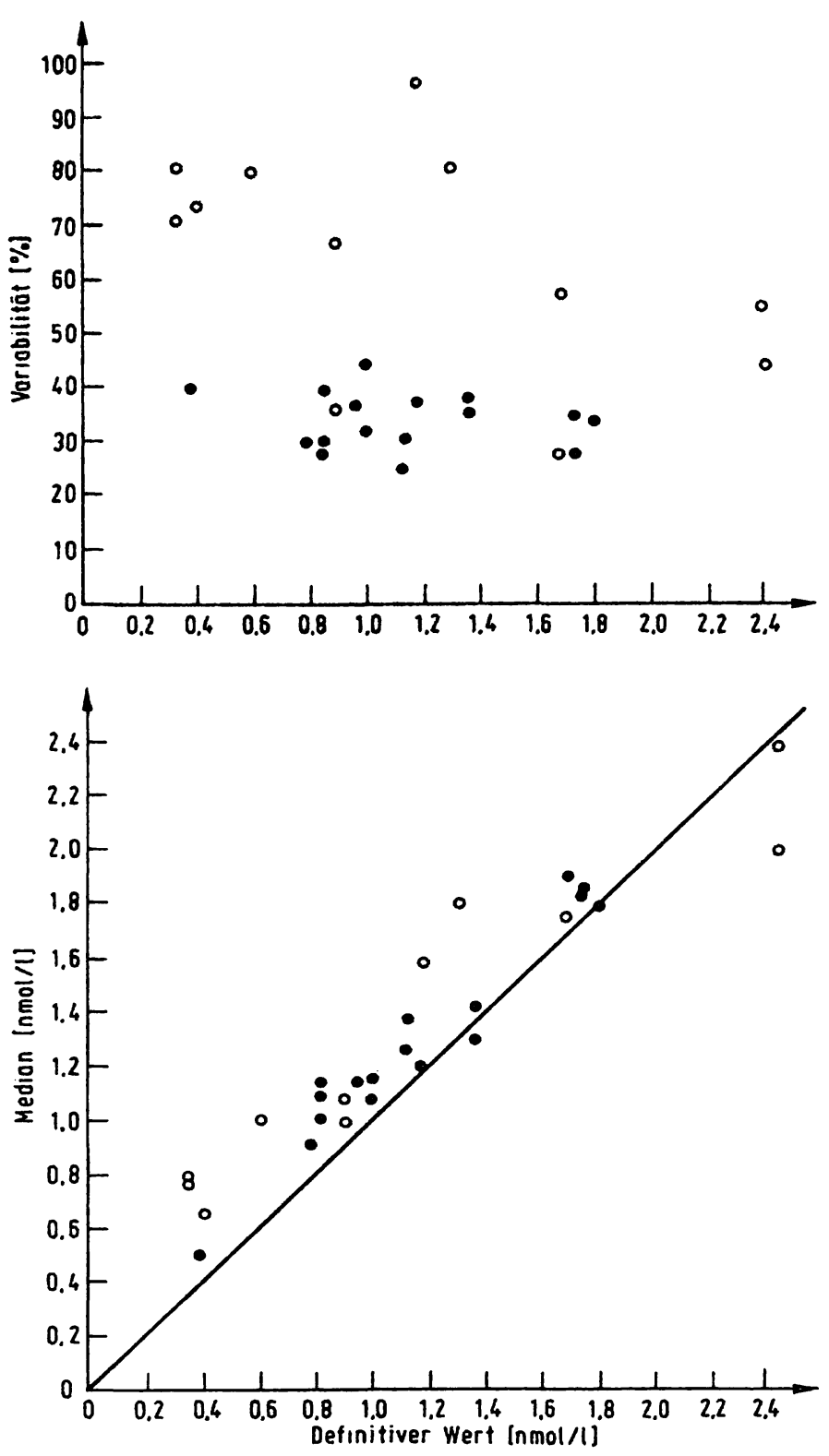

Abb. 5. Relative Variabilität (oben) der Ergebnisse aus 14 Ringversuchen und die Werte der Mediane (unten) - jeweils gegenüber den gegebenen Konzentrationen von Östradiol-17ß. O Gestripptes Plasma; O Pool-Plasma.

gen die Werte für die Variabilität konstant unter $50 \%$ - unabhängig von der Art des Probenmaterials. Ähnlich ist die Situation für die Richtigkeit, die seit dem 7. Ringvèrsuch in den meisten Fällẹn deutlich besser war als zuvor.

Auch bei Östriol (Abb. 6) könnte der Eindruck entstehen, in Pool-Proben seien präzisere Bestimmungen möglich als in gestripptem Plasma. Da die Variabilität aber erst seit dem 8 . Ringversuch auf Werte um $20 \%$ gesunken ist, muß angenommen werden, $\mathrm{da}$ im wesentlichen eine Verbesserung der Analytik hierzu beigetragen hat. Der Vergleich der Mediane von Östriol-Bestimmungen in Pool-Proben mit den definitiven Ergebnissen zeigt in allen Fällen mehr
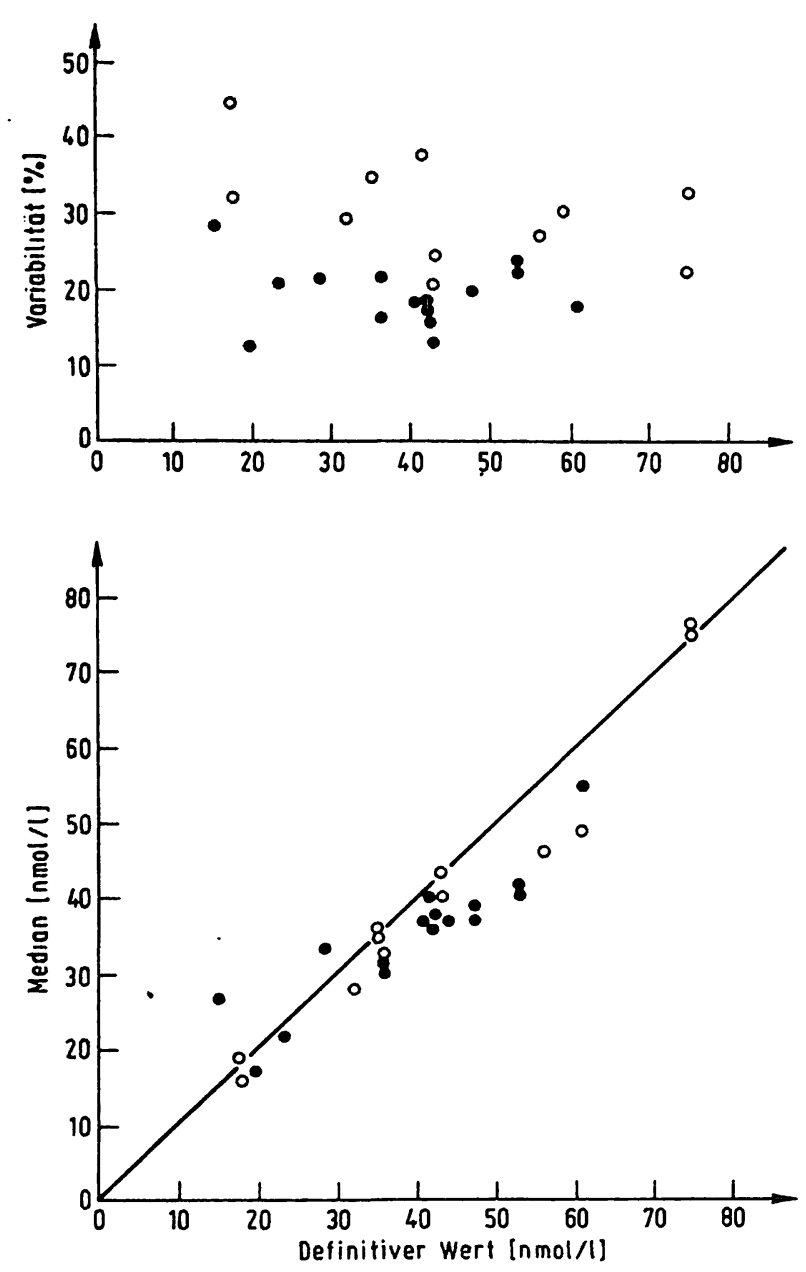

Abb. 6. Relative Variabilität (oben) der Ergebnisse aus 14 Ringversuchen und die Werte der Mediane (unten) - jeweils gegenüber den gegebenen Konzentrationen von Östriol. O Gestripptes Plasma; O Pool-Plasma.

oder weniger starke Abweichungen zu niedrigeren Werten, ohne daß eine Erklärung hierfür gegeben werden kann.

Sehr unterschiedlich hinsichtlich Richtigkeit und Präzision ist die Qualität der Bestimmungen von Progesteron (Abb. 7). Während die Úbereinstimmung zwischen Median und definitivem Wert über den gesamten Konzentrationsbereich als gut bezeichnet werden kann, ist die Präzision der Werte ähnlich wie für Aldosteron - insbesondere bei niedrigen Konzentrationen sehr schlecht. Abhängigkeiten von der Art des Probenmaterials sind nicht zu erkennen.

Die Daten für Testosteron (Abb. 8) schließlich weisen keinerlei Abhängigkeiten von den Versuchsbedingungen auf. Abgesehen von wenigen Ausnahmen - liegt der Wert für die Variabilität während des gesamten Beobachtungszeitraumes um 30\%. Die Mediane der Analysenergebnisse stimmen im allgemeinen mit den definitiven Werten gut überein. 

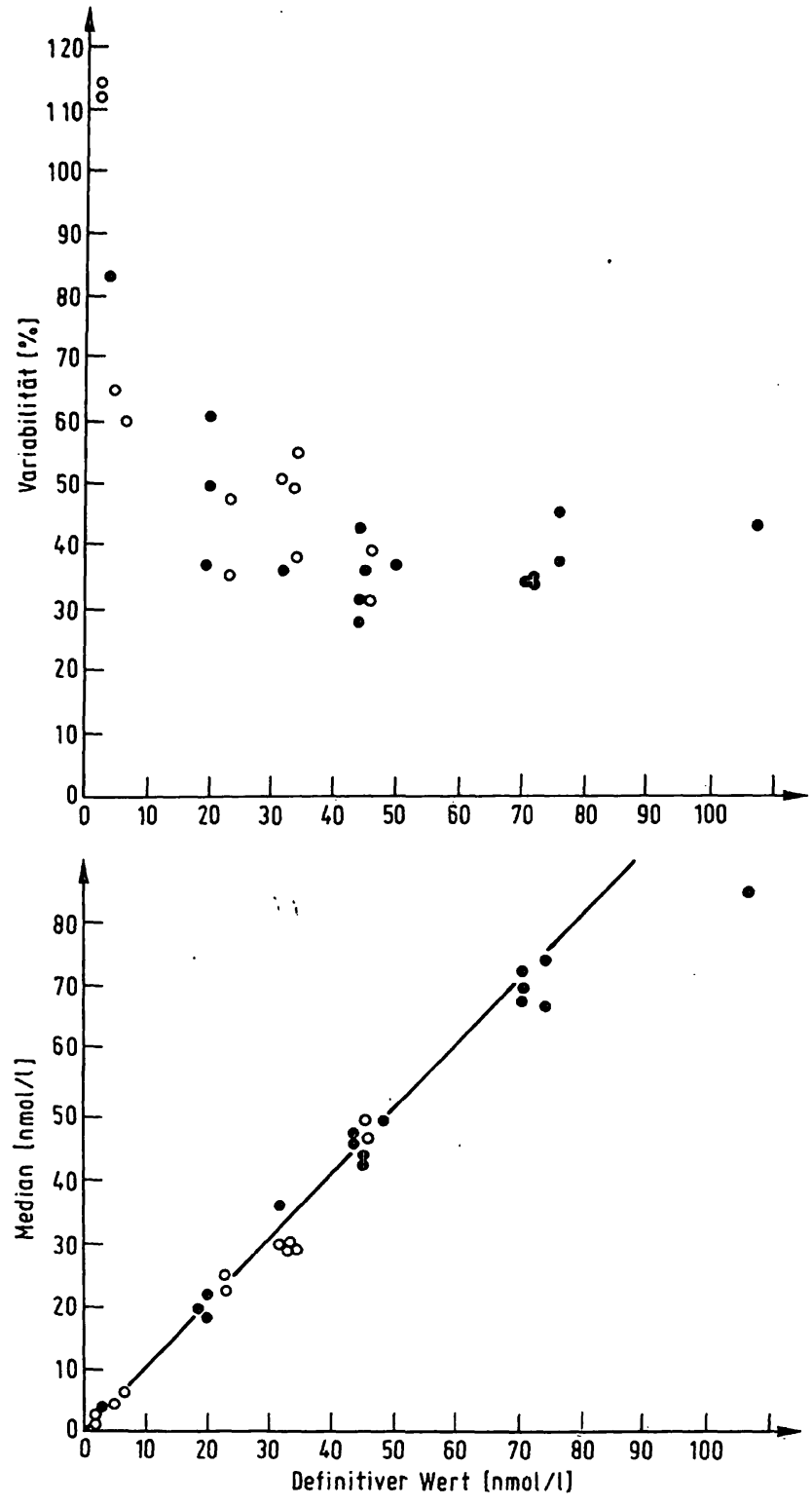

Abb. 7. Relative Variabilität (oben) der Ergebnisse aus 14 Ringversuchen und die Werte der Mediane (unten) - jeweils gegenüber den gegebenen Konzentrationen von Progesteron. O Gestripptes Plasma; O Pool-Plasma.

\section{Schlußfolgerung}

Die vorliegenden Ergebnisse von insgesamt 14 Ringversuchen während der letzten fünf Jahre lassen den Schluß zu, daß die Zuverlässigkeit der Bestimmungen von Steroidhormonen im Vergleich zu anderen klinisch-chemischen Kenngrößen sehr schlecht ist. Für einige Steroidbestimmungen ist immerhin eine Verbesserung der Präzision und der Richtigkeit während des Beobachtungszeitraumes zu erkennen. Unter Vernachlässigung der extrem ungünstigen Resultate von Aldosteronbestimmungen kann aber auch festgestellt werden, daß es grund-
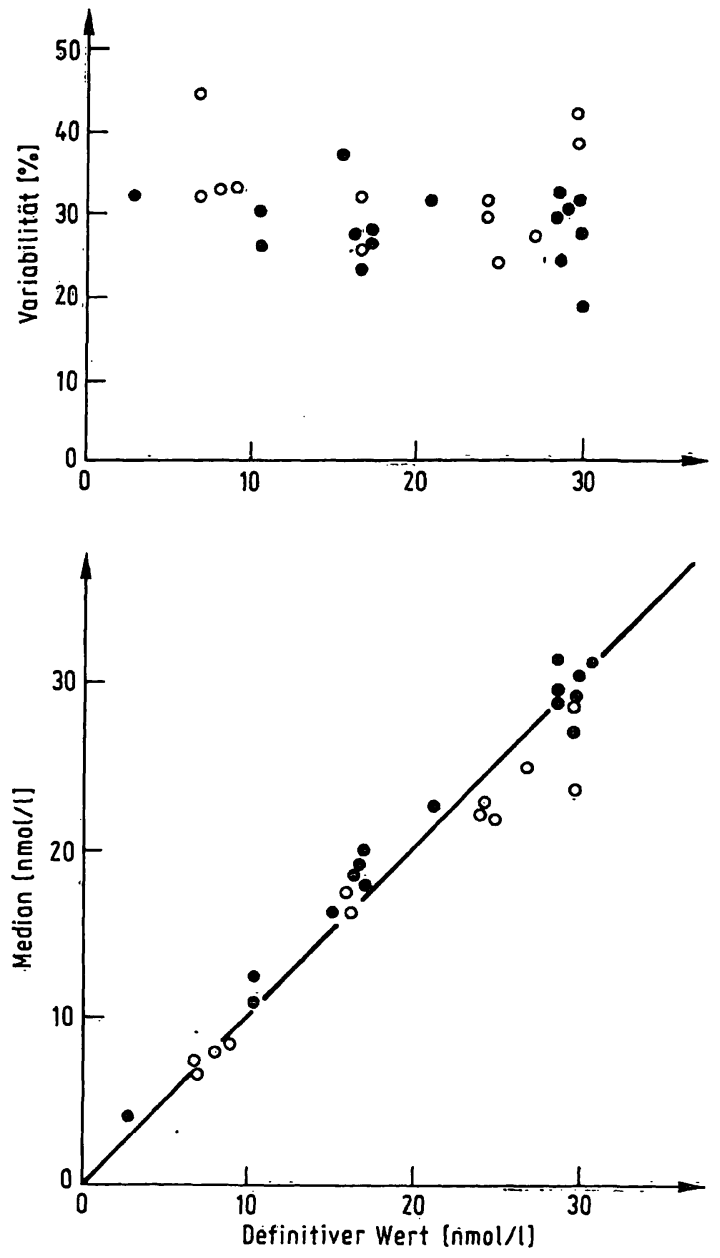

Abb. 8. Relative Variabilität (oben) der Ergebnisse aus 14 Ringversuchen und die Werte der Mediane (unten) - jeweils gegenüber den gegebenen Konzentrationen von Testosteron. O Gestripptes Plasma; O Pool-Plasma.

sätzlich möglich ist, die richtigen Werte zu ermitteln; dies zeigt die in vielen Fällen gute Ubereinstimmung von Median und definitivem Wert. Falls es gelingt, spezifischere Antikörper żu erzeugen, müßte eine weitere Verbesserung der Richtigkeit möglich sein.

Das Hauptproblem liegt offenbar in der unzureichenden Vergleichbarkeit von Analysenergebnissen aus verschiedenen Laboratorien. Von einer möglichen Standardisierung der Methoden können nach der bisherigen Erfahrung keine entscheidenden.Verbesserungen erwartet werden, da die Analysenergebnisse von Benutzern gleicher Kits - trotz nahezu gleicher analytischer Voraussetzungen - keine signifikant bessere Präzision aufweisen als die Gesamtergebnisse (13). Andererseits werden bei der laborinternen Präzisionskontrolle meist sehr gute Ergebnisse erzielt. Man kann also davon ausgehen, daß systematische Fehler innẹrhalb der Laboratorien hauptsächlich zu der unzureichenden Vèrgleich- 
barkeit der Werte verschiedener Laboratorien führen. Diese systematischen Fehler könnten erkannt und weitgehend vermieden werden, wenn den Laboratorien für die Richtigkeitskontrolle Kontrollproben mit methodenunabhängigen Sollwerten (Referenzmethodenwerte oder definitive Werte) zur Ver- fügung stünden. Die Entwicklung und Bereitstellung solcher Kontrollproben zur internen Qualitätskontrolle ebenso wie die regelmäßige Durchführung von Ringversuchen dürften daher wesentlich zur Verbesserung der Analytik von Steroidhormonen beitragen.

\section{Literatur}

1. Lawson, A. M., Mitchel, F. L. \& Breuer, H. (1977) Clin. Chem. 23, 916.

2. Marschner, I., Erhardt, E. W. \& Scriba, P. C. (1976) J. Clin. Chem. Clin. Biochem. 14, 345-351.

3. Horn, K.. Marschner, I. \& Scriba. P. C. (1976) J. Clin. Chem. Clin. Biochem. 14, 353-360.

4. Marschner, 1., Bottermann, P., Erhardt, F., Linke, R., Löffler, G.. Maier, V.. Schwandt, P., Vogt, W. \& Scriba, P. C. (1974) Horm. Metab. Res. 6, 293-296.

5. Wood, W. G., Bauer, M., Marschner, L. \& Scriba, P. C. (1980) J. Clin. Chem. Clin. Biochem. 18, 183-192.

6. Breuer, H.. Jungbluth, D., Marschner, I., Röhle, G., Scriba, P. C. \& Wood, W. G. (1978) in: Radioimmunoassay and Related Procedures in Medicine 1977, Vol II, International Atomic Energy Agency, Vienna, pp. 81-89.

7. Röhle, G. \& Breuer, H. (1978) Horm. Res. 9, 450-454.

8. Röhle, G., Jungbluth, D. \& Breuer, H. (1979) in: Biologic Prospective-1978-Pont-A-Mousson, Masson, Paris, New York, Barcelona, Milan, pp. 856-861.

10. Breuer, H. \& Röhle, G. (1981) Schweiz. Rundschau Med. (Praxis) 70, 101-109.

11. Röhle, G. \& Breuer, H. (1982) Akt. Endokrinol. Stoffw. 3. $33-38$.

12. Siekmann, L. (1979) J. Steroid Biochem. 11. 117-123.

13. Breuer, H. \& Röhle, G. (1980) J. Clin. Chem. Clin. Biochem. $18,659-660$.

\section{Dr. G. Röhle}

Institut für Klinische Biochemie

- Sigmund-Freud-Straße 25

D-5300 Bonn 1 
' 